\title{
A Histogram based Algorithm for Denoising Images Corrupted with Impulse Noise
}

\author{
Jasmeen Kaur \\ Lecturer \\ RBIENT, Hoshiarpur
}

\begin{abstract}
An algorithm is designed for the histogram representation of an image, subsequent detection of Salt and Pepper (SP) noise in the image and finally the restoration of the image from this noise. The image is first represented as histogram, then fuzzy rule are applied on this. The images taken up for experimental analyses are subjected to the fuzzy based filter for SP noise removal. The proposed algorithm exhibits superiority over traditional algorithms and recently proposed ones in terms of visual quality, Peak Signal to Noise Ratio (PSNR) and Mean Square Error (MSE). This superior performance of the fuzzy based Histogram Adaptive Filter is solely due to the histogram representation of the test images.
\end{abstract}

\section{Keywords:}

HAF, PSNR, MSE, Median filter, Mamdani's algorithm

\section{Introduction}

Images are often corrupted by different types of noise. This can be any type of noise like Gaussian noise, Impulse noise, Speckle noise etc. In this paper salt and pepper noise is added to corrupt an image. The image distorted by Salt and pepper noise can corrupt pixel by either maximum or minimum gray level. Amongst these standard median filter has been established as reliable - method to remove the salt and pepper noise without harming the edge details. However, the major drawback of standard Median Filter (MF) is that the filter is effective only at low noise densities, as the noise densities start increasing, the edge details of the original image will not be preserved by standard median filter. Also images are often contaminated by factors such as channel transmission error, impulse noise from noisy sensors.[1] Since smoothening a region will destroy image details, while sharpening may lead to unwanted intensification of noise, attenuation of noise and preservation of details are usually two conflicting aspects of image processing. Thus image enhancement is a fundamental problem in image processing.[3]For image cleaning several filtering techniques are available, but sometimes one technique is not useful for all applications. To overcome these difficulties and to provide a more efficient tool for processing image, a new fuzzy based histogram adaptive filter was proposed. Firstly we apply histogram approach for noise added image. It will separate all the pixels according to their values in the histogram form. Reading the pixels value from the histogram graph, we are able to compute those pixels that have the minimum values. Then we find out the noise and noise free pixels inside the window by applying our fuzzy algorithm which replaces the noise pixels.[7] The performance of the proposed algorithm is tested for various levels of noise corruption and compared with standard filters namely standard mean filter (SMF), fuzzy filter (FF). Our proposed method performs well in removing low to medium density impulse noise with detail preservation up to a noise density of $60 \%$ and it gives better Peak Signal-to-Noise Ratio (PSNR) and Mean square error (MSE) values.

\section{METHODOLOGY}

In this paper the Fuzzy based Histogram Adaptive Filter is used to de-noise an image corrupted with salt and pepper noise. The following section describes the Histogram Adaptive Filter (HAF) and Proposed Algorithm used in the present work.

\subsection{Fuzzy Algorithm}

Fuzzy image processing is not a unique theory. It is the collection of all approaches that understands, represent and process the images, their segments and features as fuzzy sets. The representation and processing depend on the selected fuzzy technique and on the problem to be solved. Fuzzy based image processing algorithm provides promising results over classical filters. For recovering a heavily noise corrupted image, fuzzy set theory is very helpful. In this, the image is represented by a membership function and different types of fuzzy rules which by considering the neighborhood information removes the noise but preserve edges and retain smoothness. [14]Image and fuzzy set can be modeled in a similar way where the image pixels range is from $0,1,2 \ldots .255$. In this way the image is considered as a fuzzy set and thus fuzzy based algorithms are designed.

For mapping output from a given input fuzzy inference system with fuzzy logic is used. In this proposed algorithm Mamdani's type fuzzy logic is used, which is an inbuilt function in MATLAB.

The design of histogram adaptive filter used for image processing is based on fuzzy logic. It consists of mamdani fuzzy logic which has different rules. By checking the neighboring pixels and after implementing these set of rules on these pixels, the output of the pixel is then decided that whether this is a noisy picture or a healthy pixel. Following are the steps which are carried out in the sequence to get the desired output: 
1. Read the original image

2. Convert it into gray scale image if it is a RGB image

3. Add salt and pepper noise to an image

4. Calculate the size of an image

5. Now an array is already made, automatically all the pixel value of an image will be put in that pre-defined array

6. Call the fuzzy array by using command load array

7. Scan the whole image and pass the parameter to the FIS

8. Compare the enhanced image

\section{Algorithm Description}

In this proposed algorithm, two mamdani type fuzzy inference systems have been made, namely: fuzzy noise fis and layer 2 fis. These two fis in detail are discussed below:-

\subsection{Fuzzy noise and layer 2 fis}

In fuzzy noise fis editor two inputs are taken namely inp1 and inp2, which further gives single output named as out. For input five membership functions were choosen i.e. very low, low, med, high and very high, with range of -255 to 255 . For defining these membership functions trapmf type was chosen. Similarly for the output 0 to100 range was selected with two membership functions i.e. low and high. For this fis rules are built in rule editor.

Now the output of fuzzy noise fis, is considered as input for layer 2 fis. This fis has again two inputs and has two membership function low and high. The reason behind choosing only two membership function is, as the pixel can have only two values i.e 0 or 1 , so these two membership functions specify the value of these pixels.

\section{Histogram Adaptive Filter}

When an image is interfered by salt and pepper noise, there will appear some dark spots and bright spots on the image, which seriously affect image's quality. Thus filtering out these spots to get a clear image is very important task in image processing.[8] Although other filters are also proposed for removing such type of noise, but somehow they suffer from inability to remove those dark spots and bright spots on the image simultaneously. Hence there feasibility is poor. So here a fuzzy based histogram adaptive filter is introduced, which performs fuzzy processing to the histogram of a original image. The advantage of using this type of filter is that, it utilizes information of original image's histogram and its start up time is shorter.[11] Histogram is a technique used to improve contrast in grayscale images. Figure 6.4 shows the intensity histogram for the image from figure 6.3. The histogram provides a convenient summary of the intensities in an image, but is unable to convey any information regarding spatial relationships between pixels. In this example, image does not contain many very low or very high intensity pixels. It is possible that peaks in the histogram correspond to objects in the image, but it is difficult to be certain without visually examining the image.

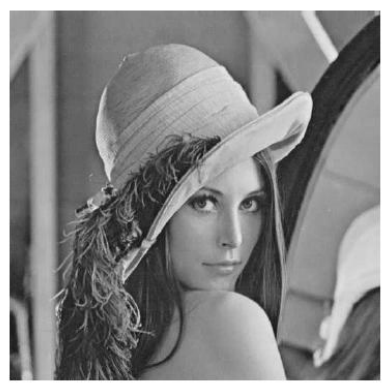

Fig.1. Gray Scale Lena Image of Resolution $512 \times 512$

The proposed fuzzy based histogram adaptive filter (HAF) is particularly effective for removing salt and pepper noise while preserving edge sharpness. This is accomplished through a fuzzy smoothing filter constructed from a set of fuzzy IF-THEN rules, which alternate adaptively to minimize the output mean squared error as input histogram statistics change. An algorithm is developed to utilize input histogram to determine parameters for the near-optimal fuzzy membership functions. Construction of the HAF filter involves three steps:

1. Define fuzzy sets in the input space,

2. construct a set of IF-THEN rules by incorporating input histogram statistics to form the fuzzy membership functions, and

3. Construct the filter based on the set of rules.

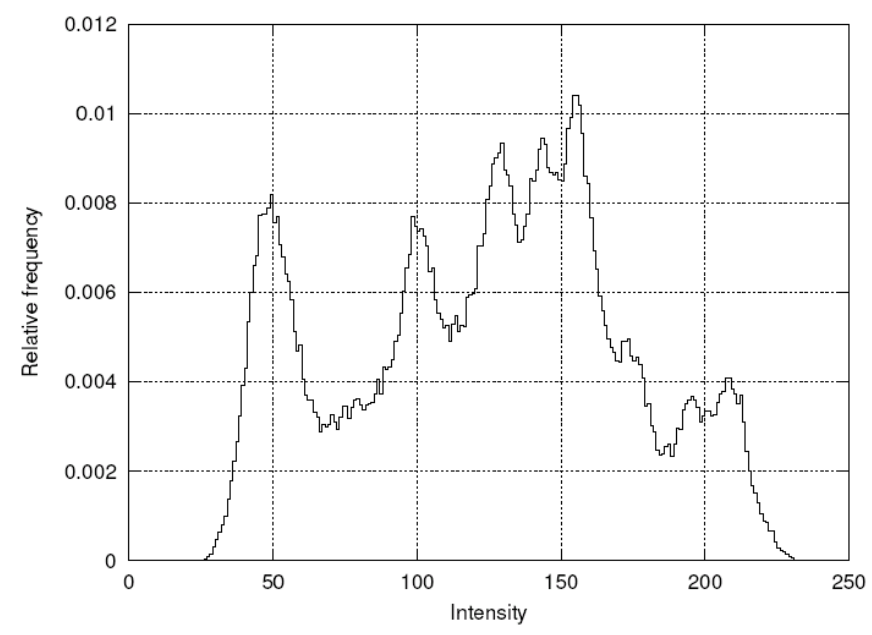

Fig.2. the Histogram of Gray Scale Lena Image 512x512 


\section{Experimental Results}

The designed filter was tested using several noise percentages. The original image is corrupted by salt and pepper noise. The $P S N R=10 \log _{10} \frac{255^{2}}{M S E}$ filter was trained using original Lena image and the noise corrupted image. The values of MSE (mean square error) and PSNR (peak signal to noise ratio) of the proposed filter is compared to the other filters. The MSE, PSNR have been computed as

$$
M S E=\frac{1}{N^{*} M} \sum_{i=1}^{N} \sum_{j=1}^{M}\left(f^{\wedge}(x, y)-f(x, y)\right)^{2}
$$

Table 1. Results in PSNR for the pepper.jpg image at various noise levels for proposed algorithm

\begin{tabular}{|c|c|c|c|c|}
\hline \multirow{2}{*}{ Noise } & \multicolumn{3}{|c|}{ Peak Signal to Noise Ratio (PSNR) in decibels (dB) } \\
\cline { 2 - 5 } density \% & Noisy image & Mean filter & Fuzzy filter & Fuzzy Based \\
& & & & HAF \\
\hline 10 & 22.7174 & 30.9531 & 41.0532 & 42.6334 \\
\hline 20 & 19.7273 & 28.1027 & 37.5256 & 38.9389 \\
\hline 30 & 18.0036 & 26.3936 & 34.1382 & 37.0955 \\
\hline 40 & 16.7353 & 24.9884 & 32.7371 & 35.1694 \\
\hline 50 & 15.8178 & 24.0805 & 30.1888 & 32.9537 \\
\hline 60 & 14.9610 & 23.1624 & 29.1574 & 31.2861 \\
\hline 70 & 14.3220 & 22.4793 & 27.6087 & 30.0435 \\
\hline
\end{tabular}


Graph1. PSNR Comparison results of proposed fuzzy histogram adaptive filter for pepper.jpg $512 \times 512$ image

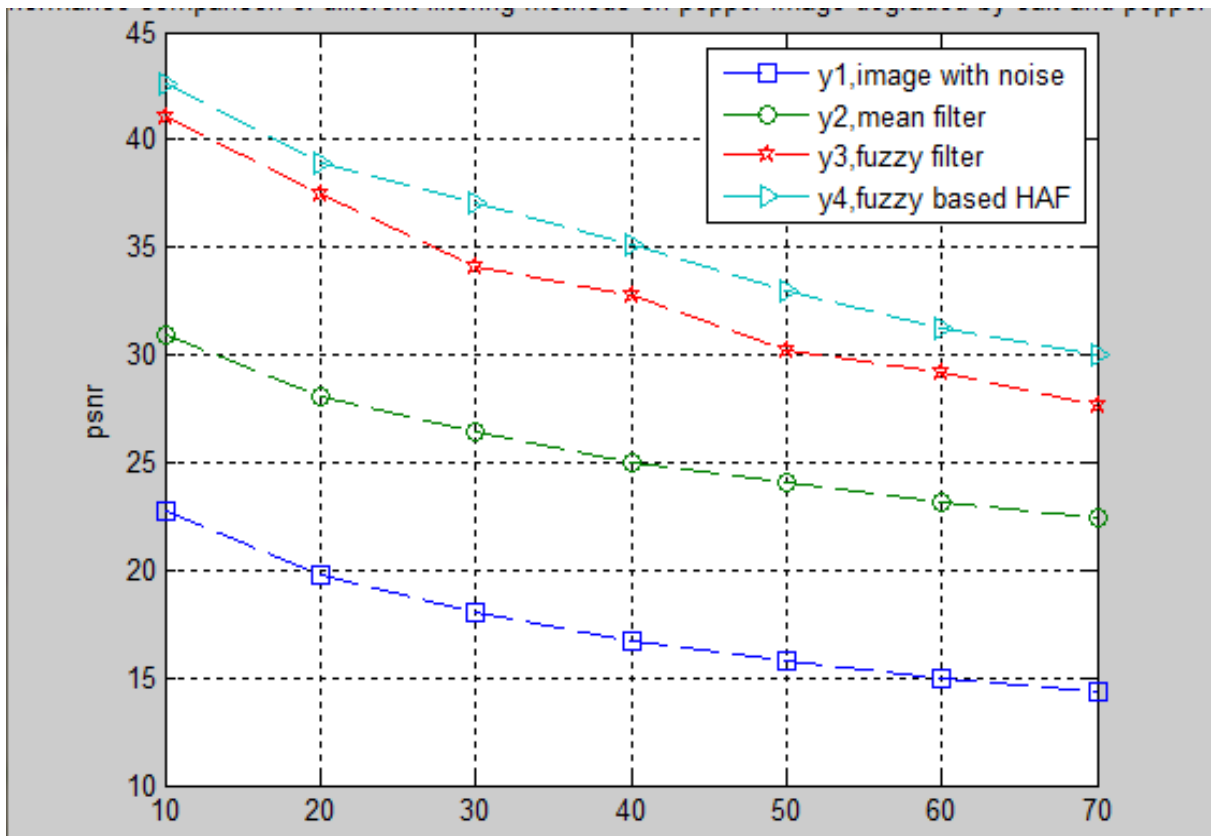

Fig.3. a) Noise free pepper image b) image with $30 \%$ of salt and pepper noise c) denoised image with mean filter d) denoised image with fuzzy filter e) denoised image with fuzzy HAF filter

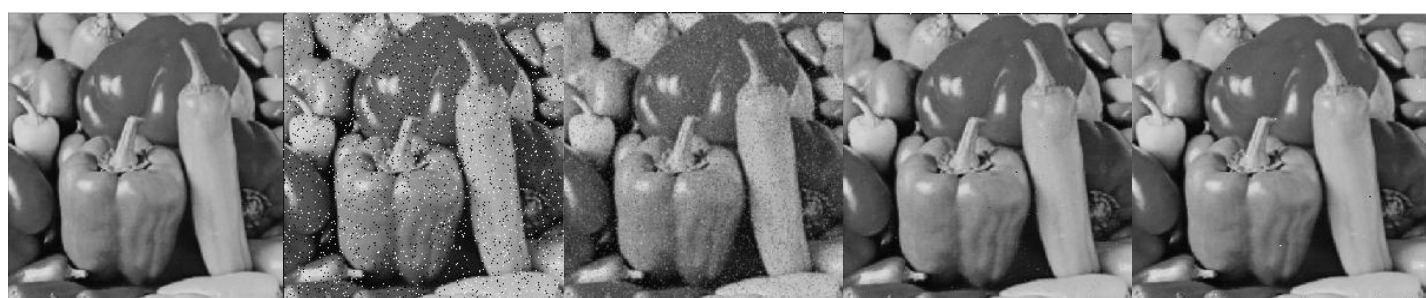

(a)

(b)

(c)

(d)

(e)

\section{Conclusion}

The working of fuzzy based histogram adaptive filter has been developed successful. The PSNR performance shows the largest values even at high noise percentage. In the future the existing filters can be modified by applying neuro-fuzzy techniques which can more efficiently enhance the corrupted image.

\section{References}

[1] J.Alex Stark, "Adaptive Image Contrast Enhancement using Generalization of Histogram Equalization", IEEE transactions on Image Processing Vol. 9, No. 5, May 2000.

[2] Rafael C.Gonzalez, Richard E.Woods, "Digital Image processing”, $2^{\text {nd }}$ edition, Prentice Hall, 2002.

[3] M.Abdullah-Al-Wadud, Md.Hasanul Kabir, M.Ali Akber Dewan, Oksam Chae, “A Dynamic Histogram Equalization 
for Image Contrast Enhancement", IEEE Transactions, Consumer Electronics, Vol. 53, No.2, pp 593-600, May 2007.

[4] Russo, F., "A new class of fuzzy operators for image processing: design and implementation", Proceedings of the Second IEEE International Conference on Fuzzy Systems, FUZZ-IEEE '93, San Francisco, March 28 - April I, pp. 815820, 1993.

[5] T. Peli, J.S. Lim, "Adaptive Filtering for Image Enhancement", Optical Engineering, v01.21, pp. 108-1 12, Jan/Feb 1982.

[6] Z.Chi,H.Yan,T.Pham, "Fuzzy Algorithms: with Application to Image Processing and Pattern Recognition", Advances in Fuzzy Systems- Application and Theory, Vol.10, 1996.

[7] Tinku Acharya, Ajoy.K.Ray, "Image Processing-Principles andApplications", Hoboken, New Jersey, A JOHN WILEY \& SONS, MC. , Publication, 2005.

[8] Detail information about the Peak signal to noise ratio, http://en.wikipedia.org/wiki/Peak_signal-to-noise_ratio
[9] Standard original images, http://www.ece.rice.edu/ wakin/images/

[10] Lee C.S., Kuo Y.H. \& Yu P.T., "Weighted fuzzy mean filters for image processing", in Fuzzy Sets and Systems, Vol. 89, 1997, pp. 157-180.

[11] Arakawa K., "Fuzzy rule-based image processing with optimization", in Fuzzy Techniques in Image Processing (Kerre E.E. \& Nachtegael M.,editors), Springer-Verlag, pp.222-247, 2000.

[12] A.K.Jain, Fundamentals of, "Digital Image Processing". Prentice-Hall, 1989.

[13] C.S.Lee,Y.H,Kuo and P.T.Yu, "Weighted Fuzzy Mean Filter for Image Processing”, Fuzzy Set Syst.,no.89, pp.157$180,1997$.

[14] F.Farbiz and M.B.Mehnaz, "Fuzzy Techniques in Image Processing” Springer-Velag, Vol.52, pp.194-221, 2000. 